\title{
Repeated nationwide point-prevalence surveys of antimicrobial use in Swedish hospitals: data for actions 2003-2010
}

\author{
G Skoog $^{12}$, J Struwe ${ }^{1}, 0$ Cars ${ }^{1}$, H Hanberger ${ }^{3}$, I Odenholt ${ }^{4}$, M Prag ${ }^{5}, K_{\text {K Skärlund }}{ }^{1}$, P Ulleryd ${ }^{67}$, M Erntell $^{8}$ \\ 1. Public Health Agency of Sweden, Solna, Sweden \\ 2. Department of Medicine Solna, Division of Infectious Diseases, Karolinska Institute, Stockholm, Sweden. \\ 3. Department of Infectious Diseases, University Hospital Linköping, Linköping, Sweden \\ 4. Infectious Diseases Research Unit, Department of Clinical Sciences, Lund University, Malmö, Sweden \\ 5. Department of Infectious Diseases, University Hospital Örebro, Örebro, Sweden \\ 6. Department of Communicable Disease Control, Region Västra Götaland, Sweden \\ 7. Department of Infectious Medicine, Institute of Biomedicine, Sahlgrenska Academy, University of Gothenburg, Sweden \\ 8. Department of Communicable Disease Control, County Council, Region Halland, Sweden
}

Correspondence: Gunilla Skoog (gunilla.skoog@folkhalsomyndigheten.se)

Citation style for this article:

Skoog G, Struwe J, Cars O, Hanberger H, Odenholt I, Prag M, Skärlund K, Ulleryd P, Erntell M. Repeated nationwide point-prevalence surveys of antimicrobial use in Swedish hospitals: data for actions 2003-2010. Euro Surveill. 2016;21(25):pii=30264. DOI: http://dx.doi.org/10.2807/1560-7917.ES.2016.21.25.30264

This study sought to analyse antimicrobial pressure, indications for treatment, and compliance with treatment recommendations and to identify possible problem areas where inappropriate use could be improved through interventions by the network of the local Swedish Strategic Programme Against Antibiotic Resistance (Strama) groups. Five point-prevalence surveys were performed in between 49 and 72 participating hospitals from 2003 to 2010. Treatments were recorded for 19 predefined diagnosis groups and whether they were for community-acquired infection, hospital-acquired infection, or prophylaxis. Approximately one-third of inpatients were treated with antimicrobials. Compliance with guidelines for treatment of community-acquired pneumonia with narrow-spectrum penicillin was $17.0 \%$ during baseline 2003-2004, and significantly improved to $24.2 \%$ in 2010. Corresponding figures for quinolone use in uncomplicated cystitis in women were $28.5 \%$ in $2003-$ 2004 , and significantly improved, decreasing to $15.3 \%$ in 2010. The length of surgical prophylaxis improved significantly when data for a single dose and 1 day were combined, from $56.3 \%$ in $2003-2004$ to $66.6 \%$ in 2010. Improved compliance was possibly the effect of active local feedback, repeated surveys, and increasing awareness of antimicrobial resistance. Strama groups are important for successful local implementation of antimicrobial stewardship programs in Sweden.

\section{Introduction}

The Swedish Strategic Programme Against Antibiotic Resistance (Strama) was established during the $1990 \mathrm{~s}$ to promote rational use of antibiotics [1]. At that time in Sweden, data on antimicrobial use in hospitals was limited to statistics on the total amount of deliveries from hospital pharmacies [2]. While such data allow analysis over time and comparison of patterns of antimicrobial use among hospitals and regions, they do not include the indication for therapy and thus do not permit assessment of compliance with recommendations. Knowledge of antimicrobial use in Swedish hospitals was largely restricted to local projects [3] when the national Strama board [1] initiated the nationwide point-prevalence survey (PPS) in 2003. In a PPS, data is registered during one day and gives a cross-sectional figure over, in this case, antibiotic use for different diagnosis groups. Numbers are small when looking only at one hospital but if data are collected for several hospitals, and surveys are repeated, data becomes more reliable.

This paper presents an analysis of antimicrobial use related to diagnosis in Swedish hospitals and experiences from interventions based on findings in five PPS from 2003 to 2010.

\section{Methods}

Sweden has 21 county councils and ca 80 public acutecare hospitals, all of which were invited to participate in the studies. Participation was optional and between 19 and 21 county councils and 49 and 72 acute-care hospitals (somatic care only, that is, excluding psychiatric care) over the study years. The mean length of stay (LOS) was 5.4 days in $2003,5.3$ days in $2004,5.1$ days in 2006, 5.0 days in 2008 , and 4.8 days in 2010 [4]. 


\section{FIGURE 1}

Distribution of antibiotics given to patients in the nationwide hospital point-prevalence surveys, Sweden, 2003-2010

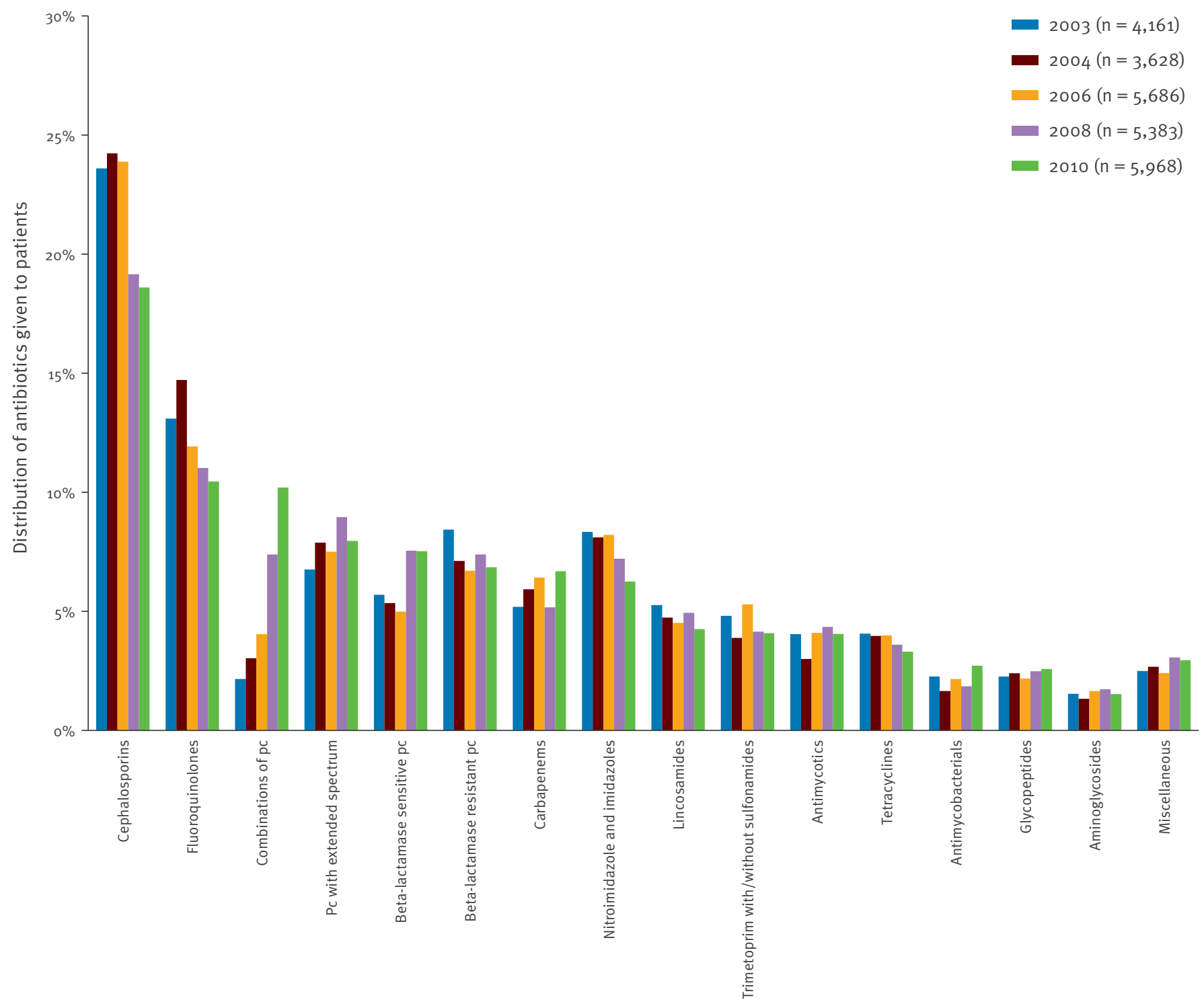

Excludes antibiotics given as prophylaxis.

The studies were performed as PPS during one day within a two-week period in November 2003, 2004, 2006, 2008, and 2010. Data were collected by infectious-disease specialists at 08:00 from the medical records of all patients receiving systemic antibacterials. The total number of admitted patients was used as the denominator. The survey protocol is available online [5]

Therapy was defined as the conjunction of the drug(s) dispensed, diagnosis and whether the prescription was for community-acquired infection (CAI), hospitalacquired infection (HAl), or prophylaxis [5]. More than one therapy could be registered for one patient, and one therapy could consist of more than one antibiotic.
The following patient data were collected: age, sex, prescribed drug(s), dose per administration, number of doses, route of administration, focus of the infection (or for prophylaxis) and significant risk factors for infection such as immunosuppression, presence of foreign material and invasive medical devices.

The assessment also included whether the indication for treatment was documented in the records and whether a relevant culture was done before initiation of treatment. A final assessment of the therapy was made by the surveyors as directed therapy, empirical therapy, or deviating from recommendations.

Duration of surgical prophylaxis was classified as single dose, 1 day, or more than 1 day. 
Distribution of indications for antimicrobial treatment according to predefined diagnosis groups, nationwide hospital pointprevalence surveys, Sweden, 2003-2010

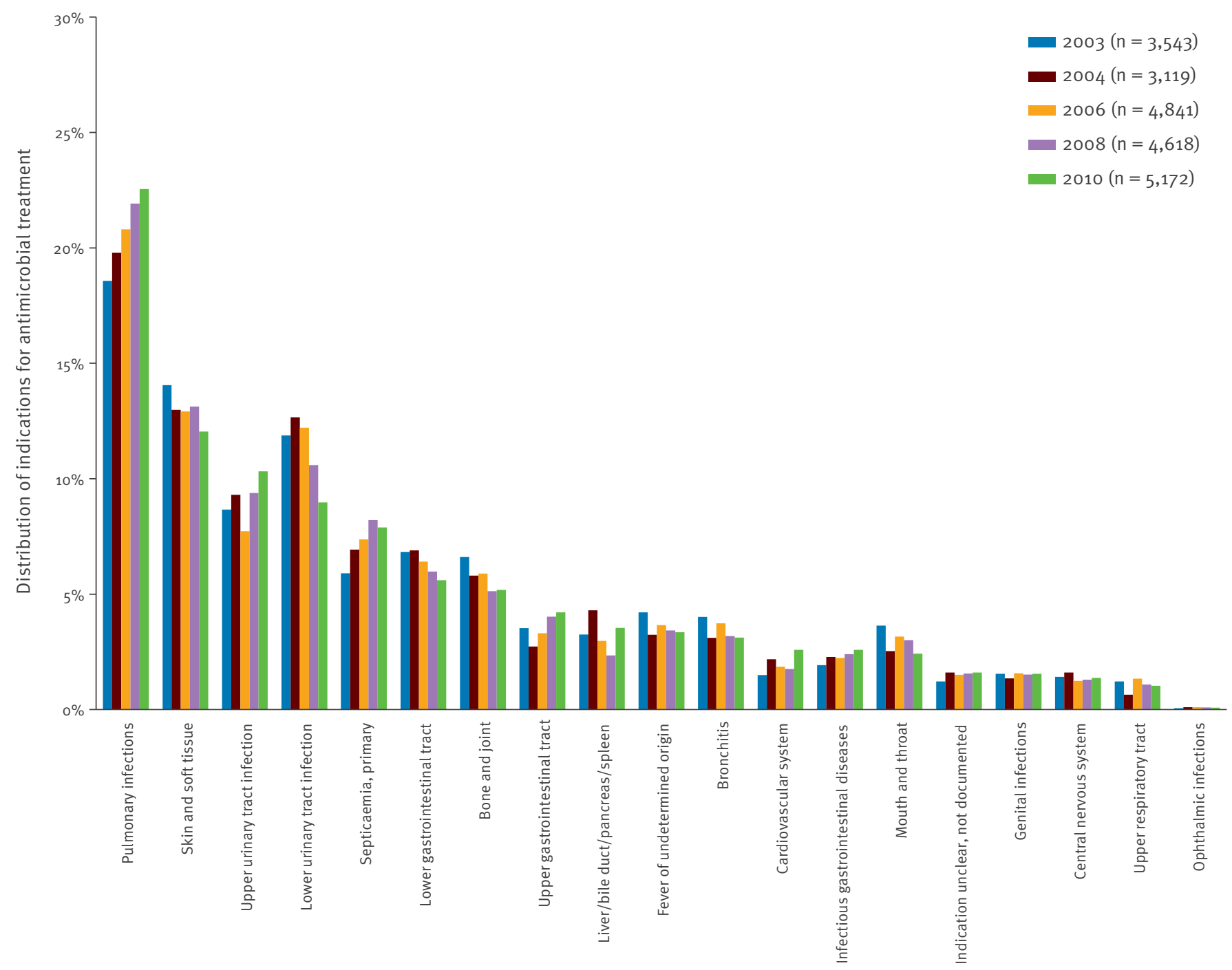

Excludes antibiotics given as prophylaxis, includes antimycotics and tuberculostatic treatments.

Antimicrobials were classified according to the World Health Organization (WHO) Anatomical Therapeutic Chemical (ATC) Classification System [6]. The defined daily dose (DDD) was used as indicator for antimicrobial pressure and calculated from the amounts of antibiotics given per day according to the WHO definitions, with the exception of oral vancomycin, for which the prescribed daily dose of $500 \mathrm{mg}$ was chosen. DDDs were not registered for children under 16 years. Drugs recorded in this study were: antibiotics (oral vancomycin (A07AA09), terbinafine (Do1BA02), oral imidazoles (P01AB) and antibacterials for systemic use (J01), excluding methenamine), antimycotics (Jo2A) and tuberculostatics (Jo4A).

Coded patient identity and study data were entered into a web-based interface (Neotide Ltd, Vaasa, Finland) by each local Strama group. A standardised validation was performed, and incongruences were addressed for reassessment by each Strama group.

A systematic approach in feeding back the results to hospitals was achieved by including the local Strama groups in the process. Together with the research team, Strama representatives were involved in formulating three main messages for interventions, based on data from the 2003 and 2004 surveys. These representatives could then present and discuss the study results in their own counties while including relevant local aspects. Interventions also took the form of information leaflets addressing main messages that were sent to all hospital prescribers and displayed in participating hospitals in visible areas such as in lifts and on bulletin boards. 
TABLE 1

Demographics in five nationwide hospital point-prevalence surveys of antimicrobial use, Sweden, 2003-2010

\begin{tabular}{|c|c|c|c|c|c|}
\hline & 2003 & 2004 & 2006 & 2008 & 2010 \\
\hline Number of available beds in Sweden & 22,471 & 22,454 & 21,628 & 21,302 & 21,019 \\
\hline \multicolumn{6}{|l|}{ Number of admitted patients in survey } \\
\hline Tertiary care hospitals & 4,808 & 3,191 & 6,675 & 5,269 & 6,849 \\
\hline Secondary care hospitals & 5,977 & 5,536 & 7,049 & 7,189 & 6,804 \\
\hline Primary care hospitals & 2,751 & 2,621 & 3,412 & 3,529 & 3,429 \\
\hline Specialised geriatric hospitals & NA & NA & NA & 331 & 621 \\
\hline Total (\% of available beds) & $\begin{array}{l}13,536 \\
(60.2 \%) \\
\end{array}$ & $\begin{array}{l}11,348 \\
(50.5 \%) \\
\end{array}$ & $\begin{array}{l}17,136 \\
(79.2 \%) \\
\end{array}$ & $\begin{array}{l}16,318 \\
(76.6 \%) \\
\end{array}$ & $\begin{array}{c}17,703 \\
(84.2 \%) \\
\end{array}$ \\
\hline $\begin{array}{l}\text { Number of patients in survey with antimicrobial treatment } \\
\text { (\% of admitted patients in survey) }\end{array}$ & $4,178(30.9 \%)$ & $\begin{array}{c}3,622 \\
(31.9 \%) \\
\end{array}$ & $\begin{array}{c}5,588 \\
(32.6 \%) \\
\end{array}$ & $5,339(32.7 \%)$ & $5,928(33.5 \%)$ \\
\hline \multicolumn{6}{|l|}{$\begin{array}{l}\text { Age of treated patients in survey } \\
\text { (\% of treated patients in survey) }\end{array}$} \\
\hline $0-16$ years & $266(6.4 \%)$ & $183(5.1 \%)$ & $280(5.0 \%)$ & $268(5.0 \%)$ & $336(5.7 \%)$ \\
\hline $17-60$ years & $1,102(26.4 \%)$ & $913(25.2 \%)$ & $1,439(25.8 \%)$ & $1,266(23.7 \%)$ & $1,453(24.5 \%)$ \\
\hline 61-79 years & $\begin{array}{c}1,624 \\
(38.9 \%) \\
\end{array}$ & $\begin{array}{c}1,465 \\
(40.4 \%) \\
\end{array}$ & $2,187(39.1 \%)$ & $\begin{array}{c}2,168 \\
(40.6 \%) \\
\end{array}$ & $2,326(39.2 \%)$ \\
\hline$\geq 80$ years & $1,186(28.4 \%)$ & $1,061(29.3 \%)$ & $\begin{array}{c}1,682 \\
(30.0 \%) \\
\end{array}$ & $1,637(30.7 \%)$ & $1,813(30.6 \%)$ \\
\hline \multicolumn{6}{|l|}{ Number of patients in ICUs } \\
\hline $\begin{array}{l}\text { Number of adults in ICUs with antimicrobial treatment } \\
\text { (\% of all treated adults) }\end{array}$ & $\begin{array}{c}176 \\
(4.5 \%) \\
\end{array}$ & $\begin{array}{c}154 \\
(4.5 \%) \\
\end{array}$ & $\begin{array}{c}266 \\
(5.0 \%) \\
\end{array}$ & $\begin{array}{c}227 \\
(4.5 \%) \\
\end{array}$ & $\begin{array}{c}240 \\
(4.3 \%)\end{array}$ \\
\hline $\begin{array}{l}\text { Number of children in ICUs with antimicrobial treatment } \\
\text { (\% of all treated children) }\end{array}$ & $\begin{array}{c}25 \\
(9.4 \%) \\
\end{array}$ & $\begin{array}{c}27 \\
(14.8 \%) \\
\end{array}$ & $\begin{array}{c}41 \\
(14.6 \%)\end{array}$ & $\begin{array}{c}26 \\
(9.7 \%) \\
\end{array}$ & $\begin{array}{c}49 \\
(14.6 \%)\end{array}$ \\
\hline
\end{tabular}

ICU: intensive care unit; NA: not available.

Statistical calculations of changes over time were performed only for the three intervention areas. The average percentage from the first two surveys were used as baseline measurements and counted as one time period (2003-2004). Changes over time were examined using chi-squared tests. First, a comprehensive test including all study years was performed. If the result of this test showed statistical significance ( $p<0.001)$, additional chi-squared tests in pairs were performed. Further statistical calculations were not considered appropriate in this descriptive survey because of the high coverage of admitted patients within somatic hospital care.

The survey was assessed by the Regional Ethical Review Board in Lund on 24 September 2003, registration number LU 596-03.

\section{Results}

Study demographics are presented in Table 1 . The number of admitted patients included varied between 11,348 and 17,703 and the number of patients with antimicrobial treatment varied between 3,622 and 5,928. The proportion of women varied between $48.7 \%$ and $49.9 \%$. The median age was 73 years old for women (range: 0-108 years, interquartile range (IQR): 27 years) and 70 years old for men (range: 0-108 years, IQR: 23 years).
The observed prevalence of CAI, HAI, and prophylaxis in different hospital categories is shown in Table 2. Tertiary hospitals and specialised geriatric hospitals had a higher prevalence of treatment for HAl, and tertiary hospitals also had a higher proportion of patients on medical prophylaxis compared with the other hospital categories.

The mean antimicrobial pressure within adult specialties was 40.3 DDD per 100 admitted patients in 2003, 43.1 in 2004, 43.5 in 2006, 45.9 in 2008, and 45.6 in 2010. Antibiotics represented an average of $92.1 \%$, antimycotics, $2.4 \%$, and tuberculostatics, $5.5 \%$ of the total DDD per 100 admitted patients, respectively.

The documented indication for antibiotic treatment in medical records varied over the study years between $82.3-85.2 \%$. After oral completion of information from the staff, an indication for treatment could be obtained in $94.9 \%$ of patients. The distribution of all given antibiotics used for treatment of infections in adults (prophylaxis excluded) is shown in Figure 1. Cephalosporins and fluoroquinolones were the most frequently used antibiotics, although use decreased over the years of the study. The use of penicillins increased during the study period, especially penicillins in combination with piperacillin.

As shown in Figure 2, pulmonary infections were the most common of the 19 predefined diagnosis groups. 
Prevalence of community-acquired infection, hospital-acquired infection, and surgical and medical prophylaxis in five nationwide hospital point-prevalence surveys of antimicrobial use, Sweden, 2003-2010

\begin{tabular}{|c|c|c|c|c|c|}
\hline & \multicolumn{5}{|c|}{ Number of therapies (\% of admitted patients) } \\
\hline & 2003 & 2004 & 2006 & 2008 & 2010 \\
\hline \multicolumn{6}{|l|}{ Community-acquired infection } \\
\hline Tertiary care hospitals & $836(17.4 \%)$ & $535(16.8 \%)$ & $1,096(16.4 \%)$ & $850(16.1 \%)$ & $1,112(16.2 \%)$ \\
\hline Secondary care hospitals & $1,022(17.1 \%)$ & $1,048(18.9 \%)$ & $1,334(18.9 \%)$ & $1,294(18.0 \%)$ & $1,404(20.6 \%)$ \\
\hline Primary care hospitals & $446(16.2 \%)$ & $464(17.7 \%)$ & $629(18.4 \%)$ & $721(20.4 \%)$ & $724(21.1 \%)$ \\
\hline Specialised geriatric hospitals & NA & NA & NA & $52(15.7 \%)$ & $65(10.5 \%)$ \\
\hline Total & $2,304(17.0 \%)$ & $2,047(18.0 \%)$ & $3,059(17.9 \%)$ & $2,917(17.9 \%)$ & $3,305(18.7 \%)$ \\
\hline \multicolumn{6}{|l|}{ Hospital-acquired infection } \\
\hline Tertiary care hospitals & $510(10.6 \%)$ & $365(11.4 \%)$ & $841(12.6 \%)$ & $690(13.1 \%)$ & $901(13.2 \%)$ \\
\hline Secondary care hospitals & $494(8.3 \%)$ & $517(9.3 \%)$ & $657(9.3 \%)$ & $653(9.1 \%)$ & $600(8.8 \%)$ \\
\hline Primary care hospitals & $235(8.5 \%)$ & $190(7.2 \%)$ & $284(8.3 \%)$ & $285(8.1 \%)$ & $281(8.2 \%)$ \\
\hline Specialised geriatric hospitals & NA & NA & NA & $73(22.1 \%)$ & $85(13.7 \%)$ \\
\hline Total & $1,239(9.2 \%)$ & $1,072(9.4 \%)$ & $1,782(10.4 \%)$ & $1,701(10.4 \%)$ & $1,867(10.5 \%)$ \\
\hline \multicolumn{6}{|l|}{ Surgical prophylaxis } \\
\hline Tertiary care hospitals & $251(5.2 \%)$ & $189(5.9 \%)$ & $332(5.0 \%)$ & $302(5.7 \%)$ & $379(5.5 \%)$ \\
\hline Secondary care hospitals & $263(4.4 \%)$ & $195(3.5 \%)$ & $306(4.3 \%)$ & $294(4.1 \%)$ & $261(3.8 \%)$ \\
\hline Primary care hospitals & $111(4.0 \%)$ & $125(4.8 \%)$ & $158(4.6 \%)$ & $171(4.8 \%)$ & $120(3.5 \%)$ \\
\hline Specialised geriatric hospitals & NA & NA & NA & $1(0.3 \%)$ & $3(0.5 \%)$ \\
\hline Total & $625(4.6 \%)$ & $509(4.5 \%)$ & $796(4.6 \%)$ & $768(4.7 \%)$ & $763(4.3 \%)$ \\
\hline \multicolumn{6}{|l|}{ Medical prophylaxis } \\
\hline Tertiary care hospitals & $120(2.5 \%)$ & $110(3.4 \%)$ & $202(3.0 \%)$ & $178(3.4 \%)$ & $301(4.4 \%)$ \\
\hline Secondary care hospitals & $95(1.6 \%)$ & $84(1.5 \%)$ & $72(1.0 \%)$ & $97(1.3 \%)$ & $90(1.3 \%)$ \\
\hline Primary care hospitals & $13(0.5 \%)$ & $19(0.7 \%)$ & $22(0.6 \%)$ & $22(0.6 \%)$ & $28(0.8 \%)$ \\
\hline Specialised geriatric hospitals & NA & NA & NA & $3(0.9 \%)$ & $6(1.0 \%)$ \\
\hline Total & $228(1.7 \%)$ & $213(1.9 \%)$ & 296 (1.7\%) & $300(1.8 \%)$ & $425(2.4 \%)$ \\
\hline
\end{tabular}

NA: not available.

All age groups are included.

Table 3 shows the three most common diagnosis groups of CAl and the antibiotic classes used for therapy. Compliance with treatment recommendations for uncomplicated community-acquired pneumonia (CAP) improved because treatment with beta-lactamase-sensitive penicillins increased significantly from $17.0 \%$ in $2003-2004$ to $24.2 \%$ ( $p<0.001$ ) in 2010. Likewise, compliance with treatment recommendations for women with lower urinary tract infections (UTI) improved significantly. In 2003-2004, fluoroquinolones were used in $28.5 \%$ for treatment vs $15.3 \%(p<0.001)$ in 2010. Beta-lactamase-resistant penicillins (isoxazolyl penicillins) were the most commonly used drugs for skin and soft-tissue infections.

Antimicrobials were administered parenterally in $43.0 \%, 44.4 \%, 47.3 \%, 48.2 \%$, and $51.0 \%$ of all cases in each study year, respectively. The proportion of treatments with a relevant culture taken before therapy ranged between the study years from $67.7-75.4 \%$ for $\mathrm{HAl}$ and $62.1-72.6 \%$ for CAI. Before parenteral treatment, culture was recorded in a range of $69.4-78.3 \%$ of the patients and before oral treatment in $60.3-69.6 \%$ of the patients.

The most frequently used antibiotics for surgical prophylaxis over the years were beta-lactamase-resistant penicillins (average 38.4\%) and cephalosporins (average $19.4 \%)$. Surgical prophylaxis was given as a single dose in $23.7 \%, 25.5 \%, 32.4 \%, 26.4 \%$, and $32.4 \%$ of all cases; during one day in 29.3\%, 35.0\%, 36.8\%, 36.3\%, and $34.2 \%$ of all cases; and for more than one day in $47.0 \%, 39.5 \%, 30.8 \%, 37.2 \%$, and $33.4 \%$ of the cases in each study year, respectively. For a single dose, no clear pattern was shown but significant improvement was seen when data for a single dose and one day were combined, from $56.3 \%$ in $2003-2004$ ) to $66.6 \%$ ( $p<0.001)$ in 2010. Medical prophylaxis constituted on average $29.7 \%$ of all prophylaxis.

\section{Discussion}

A major finding in the PPS presented here was that approximately one-third of inpatients in acute-care hospitals in Sweden were treated with antimicrobials. We found that an increasing proportion, from 
The top six antibiotic classes used to treat the three most common community-acquired infections, nationwide hospital point-prevalence surveys, Sweden, 2003-2010

\begin{tabular}{|c|c|c|c|c|c|}
\hline & \multicolumn{5}{|c|}{ Number of antibiotics given (\%) per diagnosis } \\
\hline & 2003 & 2004 & 2006 & 2008 & 2010 \\
\hline \multicolumn{6}{|l|}{ Pulmonary infections $(n=3,738)$} \\
\hline Beta-lactamase-sensitive penicillins (Jo1CE) & $98(17.0)$ & $90(16.9)$ & $121(14 \cdot 3)$ & $208(25.5)$ & $248(24.2)$ \\
\hline Cephalosporins (Jo1DB-J01DD) & $190(33.0)$ & $197(36.9)$ & $287(33.8)$ & $219(26.8)$ & $237(23.1)$ \\
\hline Penicillins with extended spectrum (Jo1CA) & $51(8.9)$ & $50(9.4)$ & $84(9.9)$ & $92(11.3)$ & $92(9.0)$ \\
\hline Tetracyclines (J01AA) & $63(11.0)$ & $67(12.5)$ & $76(9.0)$ & $66(8.1)$ & $86(8.4)$ \\
\hline Fluoroquinolones (Jo1MA) & $28(4.9)$ & $28(5.2)$ & $56(6.6)$ & $54(6.6)$ & $63(6.2)$ \\
\hline Combinations of penicillins (J01CR) & $1(0.2)$ & $3(0.6)$ & $22(2.6)$ & $36(4.4)$ & $54(5.3)$ \\
\hline \multicolumn{6}{|l|}{ Skin and soft-tissue infections $(n=2,064)$} \\
\hline Beta-lactamase-resistant penicillins (J01CF) & $109(29.1)$ & $90(29.1)$ & $144(31.8)$ & $140(31.0)$ & $157(32.9)$ \\
\hline Lincosamides (Jo1FF) & $78(20.9)$ & $64(20.7)$ & $90(19.9)$ & $95(21.1)$ & $77(16.1)$ \\
\hline Cephalosporins (Jo1DB-J01DD) & $72(19.3)$ & $45(14.6)$ & $64(14.1)$ & $47(10.4)$ & $60(12.6)$ \\
\hline Beta-lactamase-sensitive penicillins (Jo1CE) & $34(9.1)$ & $33(10.7)$ & $41(9.1)$ & $54(12.0)$ & $54(11.3)$ \\
\hline Combinations of penicillins (Jo1CR) & $8(2.1)$ & $5(1.6)$ & $7(1.5)$ & $22(4.9)$ & $33(6.9)$ \\
\hline Fluoroquinolones (Jo1MA) & $27(7.2)$ & $36(11.7)$ & $29(6.4)$ & $31(6.9)$ & $20(4.2)$ \\
\hline \multicolumn{6}{|l|}{ Female lower urinary tract infection $(n=894)$} \\
\hline Penicillins with extended spectrum (Jo1CA) & $54(35 \cdot 3)$ & $58(35.6)$ & $94(43.3)$ & $101(56.7)$ & $97(53.0)$ \\
\hline Fluoroquinolones (Jo1MA) & $37(24.2)$ & $53(32.5)$ & $41(18.9)$ & $21(11.8)$ & $28(15.3)$ \\
\hline Nitrofurantoin (Jo1XE) & $6(3.9)$ & $8(4.9)$ & $5(2.3)$ & $21(11.8)$ & $19(10.4)$ \\
\hline Trimethoprim (Jo1EA) & $34(22.2)$ & $31(19.0)$ & $56(25.8)$ & $21(11.8)$ & $17(9.3)$ \\
\hline Cephalosporins (Jo1DB-DD) & $15(9.8)$ & $12(7.4)$ & $12(5.5)$ & $7(3.9)$ & $14(7.7)$ \\
\hline Trimethoprim with sulphonamides (Jo1EE) & $3(2.0)$ & $1(0.6)$ & $7(3.2)$ & $5(2.8)$ & $4(2.2)$ \\
\hline
\end{tabular}

Codes in parentheses are classifications from the World Health Organization Anatomical Therapeutic Chemical Classification System [6].

$30.9 \%$ to $33.5 \%$, of patients received antibiotics on the day of the survey over the study years. For comparison, the European Surveillance of Antimicrobial Consumption (ESAC) surveys, which were based on a protocol similar to ours, showed that an average of $30.1 \%$ (range 19-59\%) [7] and $29.0 \%$ [8] of the patients received antimicrobials in 2006 and 2009, respectively. Standardised PPS methods for reviewing medical information is a reliable tool to describe patterns of antimicrobial use in hospitals [7,9-13]. When data are aggregated, variation is small between the separate studies, despite the expected variation in PPS methodology [14] that was previously noted in smaller studies $[10,12]$.

We found that the prevalence of HAl treated with antimicrobials was of the same order of magnitude as reported for other European countries [15] and comparable to the $8.9-11.3 \%$ obtained in the national PPS performed twice a year since 2008 by the Swedish Association of Local Authorities and Regions (data not shown).

In our surveys, the indication for antimicrobial treatment was documented in the medical records in $82.3-$ $85.2 \%$ of the cases, which is high compared with other studies $[7,8,12]$. A documented indication for treatment is important to evaluate compliance with treatment guidelines and rational use of antibiotics [7-9,12].

When we started our surveys, recommendations for antibiotic treatment in hospitalised patients were not as standardised as for some common diagnoses treated in general practice. However, the following had been published: national recommendations for indications and duration of surgical prophylaxis since $1998[16,17]$, treatment of mild to moderately severe CAP in 2004 [18], treatment of community-acquired uncomplicated lower UTI in women in 2007 [19], and recommendations for the prevention of extended-spectrum betalactamase-producing Enterobacteriaceae in 2007 [20]. Analyses after the surveys in 2003 and 2004 showed that the proportion of women with community-acquired lower UTI treated with fluoroquinolones (24.2-32.5\%), and the overall use of cephalosporins (23.6-24.2\% of all antibiotics), particularly in adults with CAP (33.0$36.9 \%$ ), seemed too high and that the duration of surgical prophylaxis often exceeded one day. Thus, the interventions before the surveys in 2006 and onward were focused on improvement in these areas.

The surveys in 2008 and 2010 suggest that the desired improvements were achieved for UTI and CAP. Also, the proportion of lower UTIs among all treated infections decreased from a maximum of $12.7 \%$ in 2004 to $9.0 \%$ in 
2010, possibly as a result of increased awareness during the feedback process not to treat asymptomatic bacteriuria. Increased use of the combination of penicillins and beta-lactamase inhibitors was probably an effect of the desire to decrease cephalosporin and quinolone use, as recommended in the ESBL-control programme [20]. Increasing awareness of antimicrobial resistance among prescribers in Sweden might also have contributed to the decreased use of cephalosporins and fluoroquinolones. These positive changes are supported by data on hospital consumption based on pharmacy sales [2]. We used an audit and feedback approach as the main intervention. Previously it has been shown that persuasive as well as restrictive approaches may affect antibiotic use [21]. However, the context in which the evidence is to be implemented plays a crucial role in the choice of methodology [22], which we addressed since feedback was given with local data. In modern interventions the role of evidence, context and facilitation is emphasised within the framework Promoting Action on Research Implementation in Health Services (PARIHS), and should be considered for future interventions [23].

It can be argued that the analysis should be restricted only to the subset of hospitals participating in all surveys, but because the coverage was so high, this should only play a minor role.

We found that compliance with evidence-based recommendations for surgical prophylaxis was poor, since the recommendation for most surgeries is only a single dose. This needs further attention, monitoring and intervention. Even if some of the prolonged prophylaxes were misclassified and were actually early treatments, they were not documented as such and were still not in accordance with existing recommendations $[16,17]$. Interestingly, around one-third of patients receiving prophylaxis in our surveys did so for medical, not surgical, conditions, an observation that has not previously been given much attention. These findings were unexpected and need to be investigated further. Medical prophylaxis might be of particular importance for the development and selection of antibiotic resistance in immunocompromised individuals who often receive long-term prophylaxis in low doses.

Earlier studies in different settings have also pointed to the high antimicrobial pressure in hospitals and frequent inappropriate use and/or poor compliance with guidelines $[9,12,24,25]$. Several specific areas for improvement suitable for quality assessment and benchmarking have been suggested, such as the high rate of intravenous administration $[9,12,25]$, poor compliance with guidelines [9], lack of culture before treatment $[9,10]$, and prolonged surgical prophylaxis $[9,12]$. The usefulness of these indicators was further discussed in the ESAC studies $[7,8,26]$.

In our study, parenteral treatments were given in 43.0-51.0\% of the cases when excluding prophylaxis.
This can be compared with more than $60 \%$ parenteral treatment in non-Swedish hospitals in other surveys $[8,9,12]$. One problem when comparing these figures is that it was not possible to assess the time that elapsed between admission and PPS. Duration of antibiotic treatment at time of PPS is not stated in several studies, including ours. In view of the comparatively short average LOS in Sweden, less than $50 \%$ on intravenous treatment is fairly low but can probably be decreased further, as previously shown in targeted interventions [27-29]. Our finding of a slowly increasing proportion of patients receiving intravenous antibiotics was paralleled by a slow increase of the mean antimicrobial pressure of 40.3-45.6 DDD per 100 admitted adult patients and a decrease in the average LOS in Swedish hospitals. The antibiotic pressure in acute care hospitals has continued to increase as measured by sales data [2]. These findings suggest that hospitalised patients in Sweden are more ill and require more intensive treatment than in previous years. When hospitals in the Baltic region were compared with one Swedish hospital, it was suggested that patients in Sweden were more severely ill compared with the other countries [12]. This should be kept in mind when analysing the proportion of intravenous antibiotic treatments as a quality indicator for rational antibiotic use. To improve comparative data analysis in the future, it would be advantageous to document the illness score, for instance with the McCabe score.

Relevant cultures are a prerequisite for rational antibiotic use. In Swedish hospitals, cultures were drawn before treatments in $62.1-75.4 \%$ of cases depending on the type of infection. This is more than the 19.7-64.5\% reported from university hospitals in five European countries [9] but fewer than the $85 \%$ reported from Norway [10]. Cultures before treatment are becoming increasingly important for patient safety, particularly in HAI, because resistance rates are increasing and the time to adequate antibiotic treatment is the most important factor for clinical outcome in severe infections [30]. This suggests that there is room for improvement.

The strength of our PPS method was the nationwide coverage and the fact that local Strama groups were given immediate access to their own data for systematic feedback to prescribers.

Weaknesses in the PPS methodology itself [14] and differences in case mix within clinical specialties and among hospitals mean that benchmarking should be performed with caution and comparisons should be focused on the performance of the same unit over time $[8,9,12,24,25,31]$. Another disadvantage with the present PPS method is the laborious way that data are collected. Even though data from our surveys are somewhat 'old' they are still relevant for benchmarking and monitoring of compliance and treatment patterns for different diagnoses in Swedish hospitals. In contrast to other publications of PPS surveys we describe 
how data have been used for widespread coordinated national feedback and interventions which were later followed up. Furthermore, our surveys informed the ESAC surveys, particularly regarding the survey protocol, and are therefore important for comparing antibiotic stewardship over time in the European Union.

We found that the repeated nationwide PPS were successfully implemented and have contributed knowledge on indications for use of antimicrobials in Swedish hospitals. The compliance with treatment recommendations for treatment of CAP and lower UTI in women has improved after interventions, while the duration of surgical prophylaxis still seems to be too long.

National and local recommendations are a cornerstone of antimicrobial stewardship. Structures like the multidisciplinary local Strama groups are of vital importance for local feedback and implementation of treatment recommendations and for the success and sustainability of antimicrobial stewardship programs. However, the laborious PPS should be replaced by IT-based systems for automatic retrieval of intention-to-treat data from digital records.

\section{Acknowledgements}

We wish to thank colleagues and staff of local Strama groups for contributing to the collection and analysis of the data as well as participating in interventions, and Anna-Maria Kling at the Public Health Agency of Sweden who performed the statistical analysis.

\section{Authors' contributions}

Otto Cars: initiated study, designed and planned the study, analysed data and planned interventions. Mats Erntell designed and planned the study, collected data, validated data, analysed data and planned interventions. Johan Struwe, Peter Ulleryd, Inga Odenholt, Mårten Prag and Håkan Hanberger designed and planned the study, collected data, analysed data and planned interventions. Katarina Skärlund and Gunilla Skoog designed and planned the study, validated data, analysed data and planned interventions. Gunilla Skoog, Johan Struwe, Håkan Hanberger and Mats Erntell wrote the manuscript.

\section{References}

1. Mölstad S, Erntell M, Hanberger H, Melander E, Norman C Skoog G, et al. Sustained reduction of antibiotic use and low bacterial resistance: 10-year follow-up of the Swedish Strama programme. Lancet Infect Dis. 2008;8(2):125-32.DOI: 10.1016/ S1473-3099(08)70017-3 PMID: 18222163

2. Public Health Agency of Sweden and National Veterinary Institute. Swedres-Svarm 2014. [Consumption of antibiotics and occurrence of antibiotic resistance in Sweden. Solna/Uppsala: 2015. Available from: http://www. folkhalsomyndigheten.se/publicerat-material/publikationer/ Swedres-Svarm-2014/

3. Struwe J, Sjögren A. [Every tenth hospitalized patient is given antibiotics for a nosocomial infection]. Lakartidningen. 2002;99(32-33):3211-3.PMID: 12219475

4. The National Board of Health and Welfare. Statistical Database, In-patient Care Diagnoses. Stockholm: Socialstyrelsen. [Accessed 25 June 2014]. Available from: http://www.socialstyrelsen.se/statistics/statisticaldatabase/ inpatientcarediagnoses
5. Swedish Strategic Programme Against Antibiotic Resistance (Strama). Survey protocol in nationwide hospital pointprevalence surveys in Sweden, 2003-2010. [Accessed 22 June 2016]. Available from: http://strama.se/ examples-of-projects/?lang=en

6. World Health Organization Collaborating Centre for Drug Statistics Methodology. ATC/DDD Index. Oslo: Norwegian Institute of Public Health. Updated 19 Dec 2013. Available from: http://www.whocc.no/atc_ddd_index/

7. Ansari F, Erntell M, Goossens H, Davey P. The European surveillance of antimicrobial consumption (ESAC) pointprevalence survey of antibacterial use in 20 European hospitals in 2006.Clin Infect Dis. 2009;49(10):1496-504.DOI: 10.1086/644617 PMID: 19842976

8. Zarb P, Amadeo B, Muller A, Drapier N, Vankerckhoven V, Davey P, et al. , ESAC-3 Hospital Care Subproject Group. Identification of targets for quality improvement in antimicrobial prescribing: the web-based ESAC Point Prevalence Survey 2009.J Antimicrob Chemother. 2011;66(2):443-9.DOI: 10.1093/jac/dkq430 PMID: 21084362

9. Vlahović-Palcevski V, Dumpis U, Mitt P, Gulbinovic J, Struwe J, Palcevski G, et al. Benchmarking antimicrobial drug use at university hospitals in five European countries. Clin Microbiol Infect. 2007;13(3):277-83.DOI: 10.1111/j.14690691.2006.01613.x PMID: 17391382

10. Berild D, Ringertz SH, Lelek M. Appropriate antibiotic use according to diagnoses and bacteriological findings: report of 12 point-prevalence studies on antibiotic use in a university hospital.Scand J Infect Dis. 2002;34(1):56-60.DOI: 10.1080/00365540110076912 PMID: 11874166

11. Andersen BM, Ringertz SH, Gullord TP, Hermansen W, Lelek $M$, Norman BI, et al. A three-year survey of nosocomial and community-acquired infections, antibiotic treatment and re-hospitalization in a Norwegian health region. J Hosp Infect. 2000;44(3):214-23.DOI: 10.1053/jhin.1999.0677 PMID: 10706805

12. Dumpis U, Gulbinovic J, Struwe J, Lagergren A, Griskevicius $\mathrm{L}$, Bergman U. Differences in antibiotic prescribing in three university hospitals in the Baltic region revealed by a simple protocol for quality assessment of therapeutic indications. Int J Clin Pharmacol Ther. 2007;45(10):568-76.DOI: 10.5414/ CPP45568 PMID: 17966843

13. Struwe J, Dumpis U, Gulbinovic J, Lagergren A, Bergman U. Healthcare associated infections in university hospitals in Latvia, Lithuania and Sweden: a simple protocol for quality assessment.Euro Surveill. 2006;11(7):167-71.PMID: 16966795

14. Gastmeier P, Sohr D, Forster D, Schulgen G, Schumacher M, Daschner $\mathrm{F}$, et al. Identifying outliers of antibiotic usage in prevalence studies on nosocomial infections. Infect Control Hosp Epidemiol. 2000;21(5):324-8.DOI: 10.1086/501764 PMID 10823565

15. European Centre for Disease Prevention and Control (ECDC). Annual Epidemiological Report on Communicable Diseases in Europe 2008. Stockholm: ECDC; 2008 . Available from: http:// ecdc.europa.eu/en/publications/Publications/o812_SUR Annual_Epidemiological_Report_2008.pdf

16. Medical Products Agency. Antibiotikaprofylax vid kirurgi - Behandlingsrekommendation [Antibiotic surgical prophylaxis - Treatment recommendations]. Information från Läkemedelsverket. 1998;9(7). Swedish. Available from: http://www.lakemedelsverket.se/malgrupp/ Halso---sjukvard/Behandlingsrekommendationerarkiv/Behandlingsrekommendation---arkivlista/ Antibiotikaprofylax-vid-kirurgi/

17. SBU - Swedish Council on Health Technology Assessment. Antibiotikaprofylax vid kirurgiska ingrepp, en systematisk litteraturöversikt - [Antibiotic prophylaxis during surgical procedures, a systematic literature review]. Stockholm: 2010 Report Number 200. Swedish. Available from: http://www. sbu.se/upload/Publikationer/Contento/1/Antibiotikaprofylax_ fulltext.pdf

18. Strålin KGG, Hedlund J, Lidman C, Spindler C, Örtqvist Å, Holmberg H. Handläggning av samhällsförvärvad pneumoni hos vuxna. Evidensbaserade riktlinjer från Svenska infektionsläkarföreningen. [Swedish guidelines for the management of adult patients with community-acquired pneumonia (CAP)]. Läkartidningen. 2008;105(38):2582-7. Swedish. Available from: http://www.lakartidningen.se/ OldWebArticlePdf/1/10284/LKTo838s2582_2587.pdf

19. Medical Products Agency. Nedre urinvägsinfektion (UVI) hos kvinnor - Behandlingsrekommendation [Lower Urinary Tract Infection (UTI) in women - Treatment recommendations]. Information från Läkemedelsverket.2007;18(2):8-45. Swedish. Available from: http://www.lakemedelsverket. se/malgrupp/Halso---sjukvard/Behandlings-rekommendationer/Behandlingsrekommendation---listan/ UVI---Nedre-urinvagsinfektion-hos-kvinnor/ 
20. Public Health Agency of Sweden. ESBL-producerande tarmbakterier [Extended-spectrum beta-lactamaseproducing Enterobacteriaceae]. Stockholm: 2014. Swedish. Available from: http://www. folkhalsomyndigheten.se/publicerat-material/publikationer/ ESBL-producerande-tarmbakterier/

21. Davey P, Brown E, Charani E, Fenelon L, Gould IM, Holmes $A$, et al. Interventions to improve antibiotic prescribing practices for hospital inpatients. Cochrane Database Syst Rev. 2013;4(4):CDo03543.PMID: 23633313

22. Palmcrantz S, Tistad M, Eldh AC, Holmqvist LW, Ehrenberg A, Tomson G, et al. Assessing feasibility and acceptability of study procedures: getting ready for implementation of national stroke guidelines in out-patient health care. BMC Health Serv Res. 2015;15(1):517.DOI: 10.1186/s12913-015-1177-5 PMID: 26596624

23. Rycroft-Malone J, Seers K, Chandler J, Hawkes CA, Crichton N, Allen $\mathrm{C}$, et al. The role of evidence, context, and facilitation in an implementation trial: implications for the development of the PARIHS framework. Implement Sci. 2013;8(1):28.DOI: 10.1186/1748-5908-8-28 PMID: 23497438

24. Willemsen I, Groenhuijzen A, Bogaers D, Stuurman A, van Keulen P, Kluytmans J. Appropriateness of antimicrobial therapy measured by repeated prevalence surveys. Antimicrob Agents Chemother. 2007;51(3):864-7.DOI: 10.1128/AAC.0099406 PMID: 17210766

25. Dimiṇa E, Kūla M, Caune U, Vĩgante D, Liepins $M$, Zeidaka L, et al. Repeated prevalence studies on antibiotic use in Latvia, 2003-2007. Euro Surveill. 2009;14(33):19307.PMID: 19712639

26. Amadeo B, Zarb P, Muller A, Drapier N, Vankerckhoven V, Rogues AM, et al. , ESAC III Hospital Care Subproject Group. European Surveillance of Antibiotic Consumption (ESAC) point prevalence survey 2008: paediatric antimicrobial prescribing in 32 hospitals of 21 European countries.J Antimicrob Chemother. 2010;65(10):2247-52.DOI: 10.1093/jac/dkq309 PMID: 20713405

27. Laing RB, Mackenzie AR, Shaw H, Gould IM, Douglas JG. The effect of intravenous-to-oral switch guidelines on the use of parenteral antimicrobials in medical wards.J Antimicrob Chemother. 1998;42(1):107-11.DOI: 10.1093/jac/42.1.107 PMID: 9700538

28. Mertz D, Koller M, Haller P, Lampert ML, Plagge H, Hug $B$, et al. Outcomes of early switching from intravenous to oral antibiotics on medical wards. J Antimicrob Chemother. 2009;64(1):188-99.DOI: 10.1093/jac/dkp131 PMID: 19401304

29. Sevinç F, Prins JM, Koopmans RP, Langendijk PN, Bossuyt PM, Dankert J, et al. Early switch from intravenous to oral antibiotics: guidelines and implementation in a large teaching hospital. J Antimicrob Chemother. 1999;43(4):601-6.DOI: 10.1093/jac/43.4.601 PMID: 10350396

30. Kollef MH. Broad-spectrum antimicrobials and the treatment of serious bacterial infections: getting it right up front.Clin Infect Dis. 2008;47(S1) Suppl 1;S3-13.DOI: 10.1086/590061 PMID: 18713047

31. Andersen BM, Rasch M. Hospital-acquired infections in Norwegian long-term-care institutions. A three-year survey of hospital-acquired infections and antibiotic treatment in nursing/residential homes, including 4500 residents in Oslo.J Hosp Infect. 2000;46(4):288-96.DOI: 10.1053/jhin.2000.0840 PMID: 11170760

\section{License and copyright}

This is an open-access article distributed under the terms of the Creative Commons Attribution (CC BY 4.0) Licence. You may share and adapt the material, but must give appropriate credit to the source, provide a link to the licence, and indicate if changes were made.

This article is copyright of the authors, 2016. 